Research Article

\section{High energy lasers and new applications}

\author{
Victor V Apollonov* \\ Prokhorov GPI RAS Vavilov str.38, Moscow, 119991, Russia
}

\section{Introduction}

In the beginning of 1973 in the USSR the study of possibility of LJE designing was conducted. The reflector, located in the tail of the rocket prototype, concentrated the obtained radiation in air and ensured micro-burst that the reactive thrust was created. The successful results of different models of the reflectors tests, which were at the same time the laser light receivers, had been obtained. One should note that all experiments pointed out above were conducted with the use of electric discharge $\mathrm{CO}_{2}$ - lasers with power up to $10 \mathrm{~kW}$, while for the injection into orbit of different highly and technologically effective equipment (global network connections, Internet, photo-monitoring of Earth surface, debris cleaning) the radiated power substantially higher is required. Thus, for example, for SC launching with the weight $1000 \mathrm{~kg}$ the laser with power not less than $15 \mathrm{MW}$ is necessary $[1,2]$.

\section{Laser jet engine development}

The laser for this application at present time can be the gas-dynamic laser (GDL) only, since only in this case the laser technology in the significant measure intersects the rocket technology, which in 50 years moved sufficiently well in the creation of super-power gas generators and it makes it possible to pose similar problems. Furthermore, the laser must work in P-P regime with the high frequency of the repetition of short pulses for the exception of the process of laser emission screening entering by the plasma, which appears with the engine work, and so for an increase in the work effectiveness [3]. In the opinion of specialists - classical missileman - LJE can find an effective use in creation of cheap single-stage means of nano- and micro-satellites launch with mass in the gap 5 - $50 \mathrm{~kg}$, which according to the opinion of experts tomorrow will compose the fundamental basis of commercial launch program. In the first stage of flight SC at the heights up to $30 \mathrm{~km}$ as the working medium in the engine the usage of atmospheric air is intended, and then to inject satellite into orbit the onboard reserve of the special fuel ablating substance- in the quantities not exceeding 15\% - 20\% of the SC started weight is needed.

\author{
More Information \\ *Address for Correspondence: \\ Victor V Apollonov, Prokhorov GPI RAS, Vavilov \\ str.38, Moscow, Russia, \\ Email: vapollo@rambler.ru; \\ vapollo@kapella.gpi.ru \\ Submitted: 27 December 2018 \\ Approved: 13 August 2020 \\ Published: 14 August 2020 \\ How to cite this article: Apollonov VV. \\ High energy lasers and new applications. \\ Int J Phys Res Appl. 2020; 3: 124-132. \\ DOI: 10.29328/journal.ijpra.1001030 \\ Copyright: @ 2020 Apollonov VV. This is \\ an open access article distributed under the \\ Creative Commons Attribution License, which \\ permits unrestricted use, distribution, and \\ reproduction in any medium, provided the \\ original work is properly cited. \\ A) Check for updates \\ (8) open access
}

The experience of powerful lasers creation is accumulated in the A. M. Prokhorov General Physics Institute of RAS and other organizations of Russia. During last few years very successful experimental studies of P-P regime in the powerful and well developed continuous laser systems were conducted. This makes it possible to approach the experimental realization of super-power P-P laser source on the basis of gas-dynamic principle and LJE in the composition of light carrier with control system. The forthcoming complex of works must become major step toward the future starting of super-lightweight SC into low near Earth orbit. The realization of project will make it possible to create highly economical LJE of reusable starting for the removal of the payloads of wide designation into space. The key advantage of new approach is connected with the fact that the source of kinetic energy and payload are united in the space and launching weight SC can be lowered to the payload weight only. An even greater $\mathrm{K}$. E. Tsiolkovskii foretold, that the starting SC of future will be achieved with the aid of the electromagnetic waves directed from the external energy source, laser at that time was not known to the World at all. The great interest of scientists and specialists, as they confirmed few last symposium in this field of research, was connected with the successful solution in our country of the problem of powerful lasers creation with high repetition rate $(50-100 \mathrm{kHz})$ of pulses of short duration (150-250 ns). This is what recently wrote "the father of laser reactive motion" Arthur Kantrowits:

"Dear Victor, thanks for the pleasure given to read your articles from the last symposium on the motion with the aid 
of laser energy which are now accessible for the scientific community. I already thought above your works and I count that these remarkable ideas on the quasi-stationary wave, about the light-detonation waves, about the matrix of reflectors those introduced into your new examination are very important for the development of theory and technology of motion with the aid of the light. With great admiration I do control the development of the laser starting in the world and in Russia in particular. I hope that after your publications the significant activity in the field of laser engine creation and its applications will be stimulated. It would be very interestingly and further hear about the progress in this hot field of research. Thanks for your energetic contribution. A. Kantrowits".

High-frequency P-P mode of laser operation is developed and tested on the base of powerful $\mathrm{CO}_{2}-\mathrm{GDL}, \mathrm{Nd}$ YAG with lamp pumping and can be successfully used for other types of the powerful lasers, such as: HF/DF, Nd YAG with LD pumping, COIL and CO. The results of computer modeling for high repetition rate P-P lasers are presented in our review. The data of modeling are dependable on the characteristics of media, way of pumping, resonator geometry, and many other parameters of the laser under consideration [4].

- COIL, P-P mode starts at frequencies > $20 \mathrm{kHz}$, Depth of modulation $-100 \%$ starts at frequencies $>100$ kHz.,Pulse duration < 250 ns. Ratio: P peak. / Paver. = $100-1000$.

- HF/DF, P-P mode starts at frequencies > $100 \mathrm{kHz}$, Depth of modulation $-100 \%$ starts at frequencies $>250$ kHz.,Pulse duration < 150 ns. Ratio: P peak. / Paver. = $1000-10000$.

- $\quad$ Nd YAG, P-P mode starts at frequencies $>4 \mathrm{kHz}$, Depth of modulation $-100 \%$ starts at frequencies $>40 \mathrm{kHz} .$, Pulse duration < 250 ns. Ratio: P peak. / Paver. $=100-1000$.

- CO, P-P mode starts at frequencies > $10 \mathrm{kHz}$, Depth of modulation $-100 \%$ starts at frequencies $>100$ kHz.,Pulse duration < 250 ns. Ratio: $\mathrm{P}$ peak. / Paver. = $100-1000$.

At present time the project of the GDL with an output power of $20 \mathrm{MW}$ with the varied high-frequency structure of emission is located in the stage of active study in our country. Meaningful results on a notable increase in the effectiveness of laser energy use in the developed mode of emission, which made it possible according to our estimations to pass from tens of kg pay-load to a hundreds, or even a thousand kg are obtained. So, one should note that the works in this region, on the basis of their large prospect for different applications, already begun in Germany, Japan, England, France, China, S. Korea, Brazil and a number of others the countries. In this case, practically all participants of that works note the chosen position of GDL as the most promising system from the point of view of its scalability on the basis of the rocket technology to the level of several ten megawatts and other parameters, important for this kind of applications.

To the present time two directions, in which the possibility of applying the laser emission in the aerospace tasks is investigated: launching to the space orbit light SC, reduction of aerodynamic drag of the flying bodies, which move in the atmosphere with the high speed were formed. In the problem of developing LJE the generated by P-P laser emission is focused by reflector near the rear end of SC and the periodically repetitive laser sparks are created. Sparks generate shock waves, which transfer the part of their mechanical pulse to the reflector, located near the tail of the rocket. As the characteristic of the previous years the gas-discharge laser systems had demonstrated the pulse repetition frequency, which was limited by the time of gas exchange in the discharge zone in the range of $100-300 \mathrm{~Hz}$. For achievement high average power - $10 \mathrm{MW}$ for this rate of operation it should be necessary to use laser pulses with the energy about $100 \mathrm{~kJ}$. With the reduced air pressure, for example for the height more than $15 \mathrm{~km}$, long living plasma sphere, created by each pulse, occupies practically entire volume of reflector, which leads to the screening of the subsequent pulses into the course $\sim$ of $10 \mathrm{~ms}$. Technical difficulty of that method of energy extraction for the laser is also a very strong impact loads with such a high energy of pulses. The use of high energy pulses with the small repetition frequency and, consequently, with the very high peak power is limited also by optical breakdown as on the route, so on the surface of reflector. The method proposed by us is the way of overcoming all that difficulties on the indicated basis: usage of laser emission with short duration of pulse and high pulse repetition frequency and mechanism of generated by OPD shock waves resonant association. Under that conditions of LJE energy of laser pulse with the short duration (100ns.) effectively (95\%) can be absorbed and converted $(\sim 30 \%)$ into shock waves. In addition to this it is shown that the specific thrust can be increased several times due to the artificial transformation of radial component of shock waves into the longitudinal one. There are many other advantages of high repetition rate P-P lasers should be mentioned here as well [5]. They are:

- Much higher efficiency of energy extraction from a media and conversion into laser light, which is important for high aperture lasers scale up;

- Plasma screening effect decreasing due to the decreasing of laser pulses duration and increasing of number of pulses up to a few tens of $\mathrm{kHz}$;

- Decreasing of laser beam thermal defocusing due to the optimal temporal structure of laser radiation;

- Localization of energy deposition in space and time and elimination of stressed state of solids under laser action due to replacement of melting by ablation; 
- Possibility of unrolled 3d geometry of breakdowns in space due to a very high repetition rate generation of laser pulses, creation of optical, acoustical and electromagnetic fields far away of laser source;

- Much longer distances of optical breakdown conditions for the same optical systems.

\section{High conductivity channel and new applications}

The displacement of the optical focusing system in the media and interaction of P-P laser radiation with energy in the focus of optical system sufficient for the breakdown ensures the formation of the continuous current-conducting channel in the air medium due to its ionization, in this case. As calculations show, the frequency band of laser pulses ensures the continuity of the formed current-conducting channel in the appropriate speed range of the moving focusing system, which can be realized in this particular medium. Actually, each pulse of the laser, with the help of focusing system can create a certain extensive region of plasma, which applies to the relatively small section of space in the trajectory of the displacements of that focusing system. If the repetition rate of pulses will follow each other with small frequency the displacements of the plasma regions after focusing system will be something more similar to the dotted line. However, with higher frequency, for example more than $10 \mathrm{kHz}$, and optimum for the medium speed the displacements of ionization regions will no longer have breaks and the formed by this process conducting channel will be continuous (Figure 1).

However, after entering of moving optical system into much less density layers of the atmosphere and further into the vacuum the problem of the scarcity of medium appears,
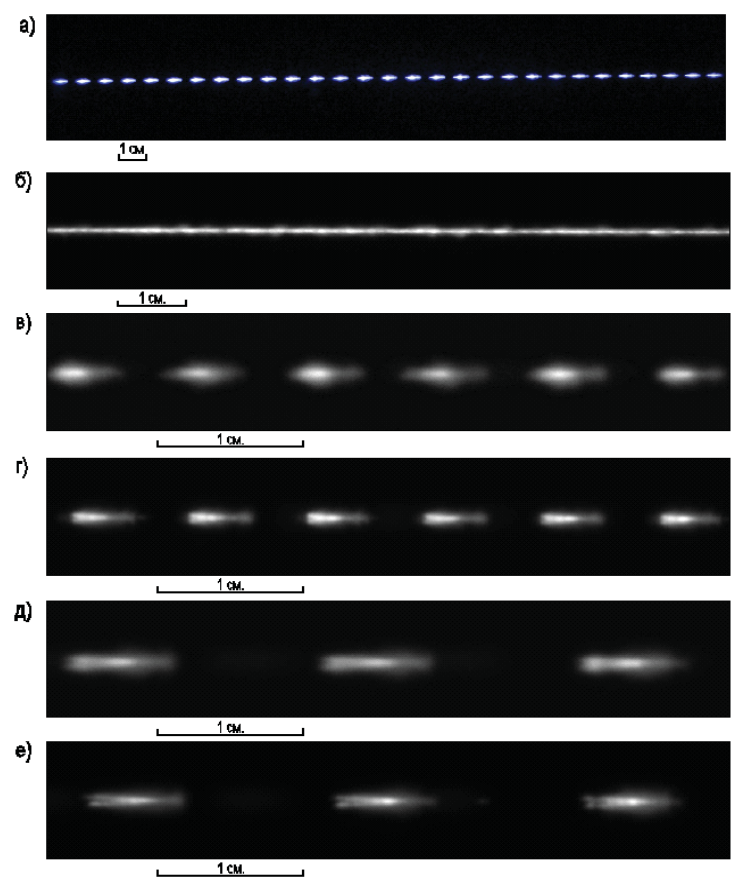

Figure 1: Time controlled evolution of trains in the space for $F=20 \mathrm{~Hz}, 50 \mathrm{~cm} / \mathrm{s}$ and for $F=25 \mathrm{kHz}, 250 \mathrm{~cm} / \mathrm{s}$ correspondingly. which could complete a deficiency in the ionized gas both as the source of conducting medium and the means of shock wave accumulation and transfer to the object of the motion of necessary quantity of energy. For this case the moving optical system must be supplied with the source of medium creation in the environments of laser focus in the form of sublimated under the action of incident laser radiation easily ionized substance, containing the nano-particles of metal or substance, which under the action of laser emission ensures the synthesis of such a nano-particles. The presence of easily ionized substance source, which due to the sublimation process under the action of laser emission, makes it possible to form the current-conducting channel in the vacuum ensures the continuity of the canal in this case. Presence of nano-particles of metal in the sublimated substance allows simultaneously with improvement of channel conductivity conditions to increase the value of the specific impulse of thrust of laser jet engine (LJE), containing mobile optical system and reservoir with the sublimated substance, which in the essence with this use has determined the time of reaching the required length of electrical power transmission channel [6].

The introduction of high voltage electrode into the currentconducting channel, formed by the moving optical focusing system, allows, from one side, to stabilize the conducting channel of the required length and direction and from the other side to transfer energy by this channel very effectively. In order to facilitate the breakdown conditions of the medium in the optical system focal point with the appearance of not conducting channel only but high energy shock wave as well, it is expedient to use the easily ionized substances, which form the steam-gas medium as the source of medium creation with low breakdown threshold. The latter fact makes it possible to decrease the requirements for the laser pulse breaking energy value.

The moving focusing system can be executed in the form the conical body or combination of cylinder and cone, inverted by apex to the side of direction of its motion. In the end of moving system special optics is established, which can be selected from the number of known optical systems, and ensuring the focusing of the laser light, which falls down to the optical system at the certain distance from the surface. In the body of moving system the sublimated under the action of laser emission easily ionized substance is placed, which contains the nano-particles of metal or substance ensuring their synthesis. As the easily ionized substance wax, paraffin, delrin (highquality acetate homopolymer) and other polymeric substances can be used. The nano-particles of metal can be of significant dimensions, for example 10-100 $\mathrm{nm}$ and can be prepared from the metals such as aluminum, tin, copper, $\mathrm{Na}, \mathrm{K}, \mathrm{Cs}$, lead and so on. The carbon-containing substances in the combination with the alkali metals can be used as the substance, which makes it possible to synthesize the nano-particles of metal or the metal-composite materials, which possess sufficiently 
high electrical conductivity. From the literature they are well known - fullerenes on the basis of alkali metals, fullerenes on the base of coppers, which demonstrate high-temperature superconductors properties with tc $>$ of $140 \mathrm{~K}$. For obtaining the nano-crystalline powder the plasma and laser methods of heating can be used. Thus, the nano-particles of carbides, oxides and nitrides with the aid of the pulse laser heating of metals in the rarefied atmosphere of methane (in the case of carbides), oxygen (in the case of oxides), nitrogen or ammonia (in the case of nitrides) were obtained. The pulse laser evaporation of metals in the atmosphere of inert gas (He or Ar) and gas- reagent $\left(\mathrm{O}_{2}, \mathrm{~N}_{2}, \mathrm{NH}_{3}, \mathrm{CH}_{4}\right)$ makes it possible to obtain the mixtures of the nano-crystalline oxides of different metals, oxcides, nitrides or carbide - nitride mixtures. Composition and the size of nano-particles can be controlled by change in pressure and composition of inert gas atmosphere and gas reagent, by power of laser pulse, temperature gradient during the cooling process. Two reasons for the fine powder to be more conductive can be taken into account. One of them is the strength of electric field increase due to the size and shape of the particles. Another one is the concentration of particles in the dust plasma track of "Impulsar" $[4,5]$. The content of nano-particles in the sublimated substance usually composes $10 \%-15 \%$ of the mass. As the source of laser emission high repetition rate P-P laser should be used. The moving focusing system is started directly from the earth's surface with the aid of high repetition rate P-P laser emission with the energy sufficient for the medium breakdown in the focus of optical system (Figure 2).

As a result of laser radiation concentration in the relatively small volume the breakdown of medium takes place. Air breakdown appears in the region of ionization, which applies to a certain distance following the moving focusing system, and the shock wave, which gives to a moving system additional momentum, leading to its acceleration. Upon the transfer of moving focusing system into rarefied layers of the atmosphere or open space, breakdown and appearing in this case shock wave will be achieved in the easily ionized substance, which the moving system has been supplied in advance. With the

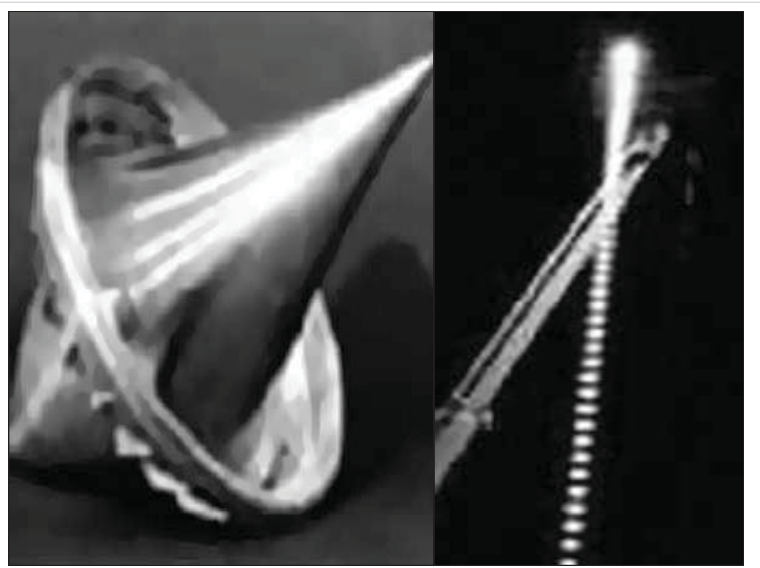

Figure 2: Launch of vehicle (SC) by low rate P-P laser (Rep. rate $-20 \mathrm{~Hz}, \mathrm{P}=10 \mathrm{~kW}$ ) arrival of the moving focusing system up to the assigned distance or on reaching by the current conducting channel the energy receiver it is necessary to place the electrode of high voltage source into the canal and to ensure the energy transfer process. The conductivity of channel proved to be sufficient for the discharge of capacitor bank to the ground just after reaching by the moving optical set of the grounded electrode, located at the final point of lift.

The results of dust plasma electrical breakdown tests are presented here without detailed explanation of physical matter of experimental arrangements. The most simple and effective dust plasma production methods were involved into our investigation. Best result of specific voltage for dust plasma breakdown was measured for electrical $\mathrm{Cu}$ wire explosion approach. The results of dust plasma modeling are [10]:

- P-P $\mathrm{CO}_{2}$ laser with train of pulses and total energy-20J. Dust particles size $50-250 \mathrm{~nm}$ and concentration $\mathrm{N}=10$ to the 10-12 power.

- For dust plasma produced by laser ablation: paraffin wax + graphite or permalloy powder $-300 v / c m$,

- For nano-particles of Al, Fe, segnetoelectrics $-350 \mathrm{v} / \mathrm{cm}$.

- $\mathrm{CO}_{2}$ single pulse laser channel, produced by conical optics - $100 \mathrm{ohm} / \mathrm{m}, 100 \mathrm{~V} / \mathrm{cm}$;

- Long channel (filaments) produced by solid state laser - 0,1 Mohm/m, 7,5kV/cm;

- Long channel produced by wire explosion - $10 \mathrm{ohm} / \mathrm{m}$, 45-50V/cm;

- Data for wire explosion channel, corresponding to the delays: $10 \mathrm{mks}$-50 mks - $75 \mathrm{mks}$; 97V/cm-124V/cm$166 \mathrm{~V} / \mathrm{cm}$.

It should be mentioned here that min. resistance value is very much dependable on cross-section of dust particles sheaf, concentration and size of particles, repetition rate of laser pulses, average power of laser, gas pressure, humidity and many other parameters are involved.

It is necessary to say several words about the interrelation of atmospheric electricity with the formation of the composition of atmosphere and climate of planet. Many years were undertaken attempt at the creation of the conducting channels of large length for studying the upper air and solution of special problems. In this connection is of great interest the program "Impulsar", which in the combination with the highvoltage high-frequency Tesla`s source can be useful in the solution of the enumerated above problems. At the same time one should say that as a result of the conducted investigations LJE is possible the passage to the solution of the following completely interesting problems: 
- Creation of the interceptor of manmade space debris and other dangerous space objects, such as asteroids, comets, meteorites and so on [9].

- Launch of micro-objects with the super-high acceleration to the space;

- Realization of orbital scale conducting channels for energy delivery from space to the ground $[10,11]$.

Powerful pulsed lasers are capable to create a large scale current conducting channels, which can be located at the arbitrary distances from the source of radiation. Channel with the length about hundreds of meters can be achieved with low energy of single laser pulse. Beginning from the 70's, successful attempts of the problems: interception of lightning bolts and blocking of over voltage waves on the electric power lines were undertaken.

\section{Lightning control}

The first and the most natural application for high power/ energy lasers is lightning! Lightning is the electrical spark discharge, which is manifested, usually, by the bright flash of light and by its accompanying thunder. The average length of lightning 2-5 km, some discharges stretch in the atmosphere up to the distance to $150 \mathrm{~km}$. Let us pause more in detail at the process of the appearance of lightning. Most frequently the lightning appears in the rain clouds, due to that they are called thunderstorm. Sometimes lightning can be formed in the layered - rain clouds, and also with the volcanic eruptions, the tornado and the dust storms. Usually are observed the linear lightning, which relate to the so-called electrode-less discharges, since they begin and end in the accumulations of the charged particles. This determines them some, until now, to not so clearly explained properties, which distinguish lightning from the discharges between the electrodes. Thus, lightning do not occur shorter than several hundred meters; they appear in the electric fields considerably of weaker than field with the inter-electrode discharges; the collection of the charges, transferred by lightning, occurs for thousands of a second from huge number of small and well isolated from each other particles, located in the volume of several $\mathrm{km}^{3}$. The process of the development of lightning in the thunderstorm clouds is most studied, in this case the lightning can pass to clouds themselves - intra-cloud lightning, and they can strike into the earth - ground-based lightning. For the appearance of lightning it is necessary that in the relatively small, but not less than the certain critical, the volume of cloud was formed the electric field with the tension, sufficient for the beginning of the electrical discharge $\sim$ of $1 \mathrm{MV} / \mathrm{m}$, and in the substantial part of the cloud there would be a field with the average tension, sufficient for maintaining the discharge $\sim$ of $0,1-0,2 \mathrm{MV} / \mathrm{m}$. In the lightning the electrical energy of cloud is converted into the thermal and the light. The process of the development of ground-based lightning consists of several stages. At the first stage in the zone, where electric field reaches critical value, begins the impact ionization, created by at first free electrons, always existing in a small quantity in air, which under the action of electric field acquire significant speeds in the direction to the earth and, colliding with the molecules, which compose air, they ionize them. Thus, appear the electron avalanches, which pass in the thread of electrical discharges - streamers, which are the well conducting channels, which, merging, give beginning to the bright thermo-ionized channel with the high conductivity - to stepped leader of lightning. Bright glow covers in this case all passed steps; then follow again stoppage and weakening of glow. These processes are repeated during the motion of leader to the earth's surface with a average speed of $200 \mathrm{~km}$. per second. The field strength at his end is strengthened in proportion to the advance of leader to the earth also under its action from the objects protruding on the earth's surface the reciprocal streamer, which is connected with the leader, is ejected. This special feature of lightning is used for creating the lightning rod. In the final stage on ionized by leader channel follow reverse (from bottom to top), or main thing, discharge of lightning, which is characterized by currents from ten to hundreds of thousands of amperes, by the brightness, which noticeably exceeds the brightness of leader, and with the high speed of advance, which at first reaches to $\sim 100 \mathrm{~km}$. per second, while in the end of that being decreasing to $\sim 10000$ kilometers per second. The temperature of channel with the main discharge can exceed $25 \mathrm{k}^{\circ} \mathrm{C}$. The length of the channel of lightning can be from 1 to $10 \mathrm{~km}$, diameter - several centimeters. After pulse advancing of ionization current of channel and its glow are weakened. In the final stage the current of lightning can last the hundredth and even tenths it flogged, reaching hundred and thousands of amperes. Such lightning are called protracted, they most frequently cause fires. Main discharge discharges the part of the cloud frequently only. The charges, located on high altitudes, can give beginning to new arrow-shaped leader, who moves continuously with a speed of thousands of kilometers in second. The brightness of its glow is close to the brightness of stepped leader. When arrow-shaped leader reaches the earth's surface, follows the second main attack, similar to the first. Usually lightning includes several repeated discharges, but their number can reach also several ten. The duration of repeated lightning can exceed $1 \mathrm{~s}$. The displacement of the channel of repeated lightning by wind creates the so-called strip lightning - luminous strip. With the entry of lightning directly into the soil is possible the formation of the unique mineral of fulgurite, which is, in essence, the sintered quartz sand. Intra-cloud lightning include usually only leader stages; their length varies from 1 to $150 \mathrm{~km}$. The portion of intracloud lightning grows in proportion to displacement to the equator, changing from 0,5 in the temperate latitudes to 0,9 in the equatorial strip. The passage of lightning is accompanied by changes in the electrical and magnetic pour on and by the radio emission, by the so-called atmospheric disturbances. The kill probability by the lightning of ground-based object grows in proportion to an increase in its height and with an 
increase in the electrical conductivity of soil on the surface or at a certain depth. The action of lightning conductor is based on these factors. If in the cloud there is an electric field, sufficient for maintaining the discharge, but insufficient for its appearance, the role of the initiator of lightning can carry out long wire cable, products of the combustion of the engine of aircraft or rocket. Specifically, thus sometimes " provoke" lightning in the layered - rain and cumulus congestus clouds.

\section{Manifestation of atmospheric electricity existence}

Why does thunder and it does sparkle lightning? In the clouds are accumulated the electric charges, which leads to the breakdown of air, which is accompanied by the flash of light and by the formation of shock acoustic wave. By other words, thunder and lightning are the manifestation of atmospheric electricity existence. The fundamental connection of thunderstorm phenomena with the electricity for two and one-half of century was reliably confirmed by numerous studies of geophysicists, meteorologists, specialists on lightning protection. However, qualitative jump in the improvement of the methods of observations in the recent two decades led to the unexpected discoveries in the study of thunderstorm clouds, lightning, orbital scale of atmospheric electrical chain. It is similar, that the splash of interest in the problems of atmospheric electricity survived now will make it necessary to glance differently at the mechanisms of shaping of electric field and its role in the dynamics of atmosphere and ionosphere of the Earth. The heart of atmospheric electrical machine - thunderstorm cloud, is more precise, the totality of simultaneously "working" thousands of thunderstorms, distributed in the lower part of the atmosphere - to troposphere. Thunderstorm cloud lives not too for long - from the hour to several hours. But for the change to some thunderstorms come others, forming in the troposphere on the proximity. Contemporary satellite measurements, and also ground-based systems of the registration of lightning give to researchers the sufficiently reliable maps of the allocation of frequencies of lightning flashes over the Earth's surface. Flash rate above the surface of ocean on the average to the order is lower than above the continents in the tropics. The reason for this asymmetry is the intensive convection in the continental regions, where the land effectively heated by solar radiation. The quick ascent of thoroughly heated saturated by moisture air contributes to the formation of powerful convective clouds of vertical development, in upper part of which the temperature lower than $\sim 40{ }^{\circ} \mathrm{C}$. In the result are formed the particles of ice, soft hail, hail, whose interaction against the background of the rapid ascending current leads to the separation of charges. Cloud height in case of big water surface in average lower than above the continents, and the processes of electrization is less effective above the ocean. In lately is discussed another factor - difference in the concentrations of the aerosols above the ocean and the continents. Since aerosols serve as the condensation nuclei, necessary for forming the particles in the supercooled air, their abundance above the land increases the probability of the strong electrization of cloud. The quantitative analysis of this factor requires detailed experiments. Approximately $78 \%$ of all lightning are recorded between $30^{\circ} \mathrm{S}$. and $30^{\circ} \mathrm{N}$. The study of the regional special features of statistics requires further improvement of the satellite and ground environment of mapping thunderstorms. these studies intensively are conducted in the connection with the realization of lightning activity as the important factor of the climate of planet, which sensitively reacts to changes in the temperature, humidity, radiation conditions and the composition of the atmosphere. The progress in studies of atmospheric electricity is connected, first of all, with the study of the mechanisms of generation and dissipation of electrical energy in the atmosphere. Main problem here - the so-called electrical dynamo: the generation of quasi-stationary electric field and space charge in the moving weakly conductive medium. Already in simplest formulation of the problem of dynamo is manifested its important special feature - very wide interval of time-spatial scales. So that on the scale in several kilometers would arise the electric field, compared in the value with the breakdown of $\sim 30 \mathrm{kV} / \mathrm{cm}$ for dry air under the standard conditions, necessary that the random traffic of charges with the collisions of cloud solid or liquid particles would lead to the matched effect of the addition of micro-currents into the macroscopic current of the completely high value (several amperes), sufficient for the rapid (tens of seconds) process of the separation of charges. It is evident from the experience that in the ripe thunderstorm cell the lightning the discharges occur with the enviable regularity - each $15-20$ s, i.e. the mechanism of charging acting in the cloud is very effective, although the average electric charge density rarely exceeds several $\mathrm{nCl} / \mathrm{m}^{3}$. As showed the measurements of electric field on the Earth's surface, and also inside the cloud medium (on the balloons, the aircraft and the rockets), in the typical thunderstorm cloud "main" negative charge - on the average several ten coulomb - occupies the interval of heights, which corresponds to temperatures from -10 to $-25{ }^{\circ} \mathrm{C}$. "Basic" positive charge composes also several ten coulomb, but it is located above basic negative; therefore the large part of the lightning discharges the cloud- earth returns to the earth negative charge. In the lower part of the cloud frequently is revealed smaller in the value the positive charge of $\sim 10 \mathrm{Cl}$. For explaining the 3-layer structure of field and charge in thunderstorm cloud described above is examined the set of the mechanisms of the separation of charges. They depend on such factors as temperature, phase composition of medium, spectrum of the sizes of cloud particles. The dependence of the value of the charge from the electric field transferred for one collision is very important. From this parameter it is accepted to subdivide all mechanisms into the induction and the noninductive. For the first class of mechanisms the charge $q$ depends on value and direction of external electric field and is connected with the polarization of the interacting particles. Non-inductive of the charge exchanges between the beings 
encountered particles in the explicit form on the field strength does not depend. In spite of the abundance of different microphysical mechanisms of electrization, now many authors consider the main thing non-inductive of charge exchanges with the collisions of small with the sizes from ones to tens of micrometers of the crystals of ice and particles of soft hail with the dimensions of the order of several millimeters. In the experiments was established the presence of the characteristic value of a temperature, at which the sign of charge q reverses, points of reverse, which lies usually between -15 and $-20{ }^{\circ} \mathrm{C}$. Specifically, this special feature made this mechanism so popular, since taking into account the typical temperature profile in the cloud it explains the 3-layer structure of the distribution of the charge density. Recent experiments showed, however that many thunderstorm clouds possess an even more complex structure of space charge. Is especially interesting mesoscale, which means the horizontal scales from ten to hundreds of kilometers, the convective systems, which serve as the important source of thunderstorm activity. Their characteristic feature is the presence of the united electrical structure, which includes the region of intensive convection and extended, sometimes to several hundred kilometers the stratified region. In the region of stratification the ascending currents sufficiently weak, but the electric field has steady multilayer structure. Near the zero isotherm here are formed sufficiently narrow, with thickness into several hundred meters, and the stable layers of space charge, mainly critical for the high lightning activity of mesoscale convective systems. A question about mechanism and laws governing the formation of the layer of positive charge in the environment of the zero isotherm remains debatable. As other examples of the work of electrical dynamo serve electrization and discharge phenomena with the dust and snowy storms, with the volcanic eruptions, nuclear explosions in the atmosphere, in the technological processes, connected with mixing of aerosol flows, for example in the flour-grinding and cement industry. The presence of powerful convective and turbulent flows in the thunderstorm clouds, and also the given above cases of electrization indicate that interaction of the large charged particles with the sizes of $0.1-10 \mathrm{~mm}$ with the particles of micrometer size in the moving conducting gas medium plays important role for the electrical dynamo. The poly-phase and multi-flow nature of medium brings in the final sum to the effective transformation of mechanical energy into the electrical. The problem of the initiation of lightning discharge remains one of sharp and unites entire complex of questions.

Briefly let us pause at two of them. First, as measurements on the balloons, the rockets and the aircraft showed, the maximum tension of electric field in the thunderstorm clouds does not exceed usually $2 \mathrm{kV} / \mathrm{cm}$, which is substantially lower than the threshold of the breakdown of dry air at the heights in question, the composing value order $10 \mathrm{kV} /$ $\mathrm{cm}$. In the thunderstorm cloud, however, the discharge is developed in the humid air, which contains the particles of different size. Natural to assume that the threshold the field is reached in the compact spatial domain, for example, on the scale less than $10 \mathrm{~m}$ or generally on the microscale because of field strengthening on the particles, and to the sufficiently short period, so that the usual sensors of field do not ensure that required three-dimensional - time resolution with the registration. The development of the avalanche of the fast, so-called running away electrons with energy on the order of $1 \mathrm{MeV}$ can serve as the alternative explanation to initiation of spark in the sufficiently weak field. Such electrons can be accelerated in the field of cloud because of a drop in brake power with an increase in the energy of particle. The threshold of the development of avalanche almost is by an order lower than the usual threshold of the breakdown of dry air; therefore when the seeding particles of the high energy are present, which are supplied by cosmic rays, the development of avalanche in the cloud can give birth to the localized region of high conductivity, capable of initiating spark. In the second place, the classical theory of gas discharge does not make it possible to explain the rapid passage of cloud medium into the conducting state at the preliminary stage of lightning. Recently the new scenario of this stage had been proposed and investigated, it is connected with the reaching by the cloud of the regime of the self-organized criticality. In the model of electrical cells with the significant dimension of $\sim 1-30 \mathrm{~m}$ and with that randomly growing in the space and the time by potential the separate small-scale breakdown between the pair of cells is capable to cause "chain reaction" of intra-cloud micro-discharges - is developed the stochastic process "of the metallization" of intra-cloud medium. This model describes well the experimentally observed special features of the preliminary stage of the lightning discharge, in that number dynamics of micro-discharges and characteristic of radio emission. The study of thunderstorm phenomena includes experiments on the artificial initiation of lightning - trigger lightning. In order to cause the discharge in the atmosphere under the thunderstorm cloud, the rocket, which leaves after itself in the regime of a deficiency in the oxidizer a significant quantity of soot conducting electricity is used. As it proved to be, initiation occurs with the sufficiently high tension of field on the earth's surface under the cloud - not less than 5-10 $\mathrm{kV} / \mathrm{m}$. The for the first time classical diagram of the initiation of discharges with the wire pulling after the rocket was realized in 1960 from onboard of research vessel. From those times about thousands of successful experiments are carried out; physics of the ascending and arrow-shaped leaders, the return shock, mechanism of the sudden strengthening of the luminosity of lightning channel because of the sharp current amplification was in detail investigated because of them. Today are opened new possibilities for control of lightning discharges, in that number with the aid of lasers. Thus, powerful lasers are capable of organizing the extensive plasma cylinders in air, which could initiate and direct the lightning discharges similar to metallic wire in the trigger lightning and 
conducting channels from the products of the combustion of special geodetic rockets. Ch. Wilson has indicated in 1925 to the possibility of accelerating the electrons to the relativistic energies in the field of thunderstorm cloud. This hypothesis was confirmed during our days: on the generation of relativistic particles and quanta of high-frequency emission in the atmosphere, which correlate with the thunderstorm activity, a whole series of experiments testifies. Thus, measurements on the balloons revealed an anomalous increase in the intensity of X-ray and gamma-radiation more than to two or three orders in the course approximately of minute with the maximum of spectrum in the region $50-60 \mathrm{keV}$. Ground observations also revealed the intensive flashes of X-radiation with the energy of quanta into several hundred $\mathrm{keV}$, whose appearance coincided with molding of the leader of lightning flash and the arrow-shaped leader of trigger lightning. Finally, for the latter several years were published the results of observations from the satellites of the splashes of the gammaquanta $\mathrm{MeV}$ - foot level, X-ray and ultraviolet radiation of atmospheric origin. For explaining these phenomena in a number of works are drawn the phenomena mentioned above new for the atmospheric electrodynamics, and precisely: the generation of the running away electrons and breakdown on the running away electrons. Energetic quanta can arise from of the bremsstrahlung of the fast electrons, which interact with the molecules of air. Models describe different situations, including the development of the avalanche of fast electrons when the extensive air shower of cosmic rays is present, and breakdown on the running away electrons in the strong field of the stepped leader of lightning. One should note that an increase in the number of fast electrons in the field of thunderstorm cloud with the presence of extensive air shower is accompanied by the generation of a large quantity of second particles, and this leads to the generation of the current pulses and radio emission. If energy of primary particle is sufficiently great $\left(10^{17}-10^{19} \mathrm{eV}\right)$, the short (several microseconds) pulse of radio emission can have the enormous energy (to $1 \mathrm{MeV}$ ), which explains the appearance of the so-called narrow bipolar pulses, observed sometimes during ground-based and satellite measurements and correlating with the thunderstorm activity. The formation of such intensive current pulses is of interest both for understanding of the mechanism of the generation of lightning and for studying the cosmic rays of super high energies. It is interesting that the presence of the cellular structure of electric field in the thunderstorm cloud with the amplitude, which exceeds the threshold "of running away", proves to be essential for the process of accelerating the electrons to the relativistic energies. The randomly oriented electrical cells together with the acceleration sharply increase the time of life of relativistic electrons in the cloud because of the diffusion nature of their trajectories. This makes it possible to explain the significant duration of the splashes of X-ray and gamma-radiation and the nature of their interrelation with the lightning flashes. The role of cosmic rays for the atmospheric electricity they must explain experiments on a study of their correlation with the studied thunderstorm phenomena.

\section{Sprites and jets investigation}

The high energy pulse to the study of atmospheric electricity at the very end XX of century gave observations of the discharge phenomena in the middle atmosphere - Sprites and Jets, which correlate with the thunderstorm activity, each day where the region of glow stretches to $85-90 \mathrm{~km}$ above the earth, and flash duration comprises from ones to tens of milliseconds, elves, who stretch to the heights 70-90 km with the duration less than $100 \mathrm{~s}$ and jet - discharges, which start from the upper part of the cloude and which are extended at times to the mesospheric heights with a speed of about $100 \mathrm{~km} / \mathrm{s}$ [12]. Registration of high-altitude discharges and diagnostics of their characteristics are complicated because of the short time of life and sporadic nature of the lightning discharges. Partly therefore, until now the physical models of Sprites, Elves and Jets remain the object of intensive discussions. Perhaps, the greatest information can be accumulated about Sprite and Jet nature and its physical examination is the most interesting application for high conductivity channel technology for the future.

The optical flash of Sprite in the mesosphere appears through several milliseconds after discharge cloud (+) - the earth, sometimes at the removal several ten kilometers along horizontal from the channel of lightning. Discharge ignites at a sufficiently high altitude, since the threshold of the breakdown of air falls with the height exponentially, whereas the amplitude of the disturbances of electric field, which appear at the moment of the powerful lightning flashes of capacitor "cloud - the earth", decreases with the height much slower according to the power law, and at the heights approximately $75 \mathrm{~km}$ in the atmosphere exceeds the threshold of breakdown. In the last decade the study of the high-altitude discharges in the atmosphere became the extensive, intensively developing direction of geophysical electrodynamics. And although the stage of the accumulation of data, that characterize the morphology of these phenomena, is by no means completed, already it is possible to switch over to a study of the thinner special features of structure and dynamics of the high-altitude discharges and their role in the orbital electrical chain and the balance of low components of the atmosphere. Experiments and theoretical calculations show that these discharges must be considered as the part of the dynamic process, which includes the formation of the structure of field and charge in the cloud and lightning discharge to the Earth. Widely are discussed different approaches to the simulation, including with the attraction of the mechanism of the running away electrons. In the detail, was developed the model of the generation of electric field in the middle atmosphere, which considers the special features of the charge distribution and its dynamics in orbital scale the convective systems, which serve as the basic sources of positive discharges to the earth. The physical model had been developed, which makes it possible to describe fine structure and dynamics of Sprite. Sprite is represented as the network of micro-discharges - 
streamers and as the self-sustaining process in the external field. Together with metallization process of thunderstorm cloud mentioned above, we have here one additional example to self-organizing, when the dynamics of high-altitude discharge is caused by reaching the threshold of the socalled directed flow, which characterizes the formation of the branched conducting channels, which overlap entire length of Sprite. Immediate prospects in the study of the high-altitude discharges in the atmosphere are connected with conducting of the coordinated ground-based and space experiments, and also with the development of the new methods of diagnostics of the parameters of high-altitude discharges, in that number with the use of possibilities of radio-wave and laser diagnostics. Electrical field of the atmosphere is very variable. The tension of the vertical component of field, which usually much more than horizontal reaches several $\mathrm{kV} / \mathrm{m}$ with the saggings, the ground-winds and the thunderstorm cloudiness. Therefore is introduced the concept of conditions grow prettier weather, that correspond to wind speed not more than $6 \mathrm{~m} / \mathrm{s}$ into the absence of any kind of sediments, hoarfrost, fog, lower cloudiness. But even under these conditions near the earth's surface there is an electric field by tension approximately $150 \mathrm{~V} / \mathrm{m}$, along which in the weakly conducting air flows the electric current with the density several $\mathrm{pa} / \mathrm{m}^{2}$. This field changes in the time and the space, the fluctuations relative to average value can comprise from ones to tens of percent. The measurements of electric field, current and conductivity under the conditions grow prettier weather they serve as the powerful means of the study of the electrical state of the atmosphere. However, to use it is possible, having only learned to divide global i.e. planetary scale, orbital - with the scale of the order of the height of neutral atmosphere $100 \mathrm{~km}$ and the local disturbances of the electrical parameters.

The latter, in their turn, are directly connected both with the changes in the ionic- aerosol composition and with the dynamics of medium. In studies it was established that the boundary layer of the atmosphere is characterized by the presence of the aeroelectrical structures, which are manifested in the pulsations of electric field with the power-law spectra short-term with the periods from ones to several hundred seconds. The electrodynamics of fog is of special interest. Full-scale observations showed that under the conditions of fog in the frequency band $10^{-2}-1 \mathrm{~Hz}$ also realize the power-law spectra of the pulsations of field, but the intensity of pulsations increases more than by the order in the comparison with the conditions grow prettier weather, which is explained by the diffusion charging of drops in the lowest layer of the atmosphere. The results it is structural temporary analysis they made it possible to isolate two forms of the electrical state of fog, the first of which is characterized by the formation of intensive aeroelectrical structures, and the second - by chaotic structural - temporary variations in the field and current.

\section{Conclusion}

Successful developments of high energy high repetition rate P-P powerful lasers technology and technology of "Impulsar" system make it possible to foresee the possibility of well conducting channels realization with the length up to several ten and hundreds of kilometers for the purpose of energy transfer for significant distances, creation of new and promising systems for the mastering of outer space power engineering and motivation for significant contribution to be done on that basis to an essential improvements of the global ecology of our planet.

\section{References}

1. VV Apollonov. High power molecular lasers, Springer. 2016.

2. VV Apollonov. To the space by laser light. Vestnik of RANS 1. 2008.

3. VV Apollonov. Yu S, Vagin VI Kislov. Conducting channel creation in a non-conductive media. RF Patent № 2009118874. 2009.

4. VV Apollonov. Yu S, Vagin VI Kislov, Patent РФ № 2175159.

5. VV Apollonov. High power lasers for "Lightcraft" applications. Proceedings of ISBEP-6. Santa Fe, (NM), USA. 2009.

6. VV Apollonov, Yu S, Vagin VI Kislov. High-frequency pulse-periodic mode for high-power laser. Proc. Of ISBEP-2, Sendai, Japan. 2003.

7. VV Apollonov. Vpered k Tciolkovskomu. Moscow. RT Soft. 2010.

8. VV Apollonov, Sorochenko VR, Yu A Shakir. Experimental investigation of resonance single wire line of energy transfer. Report of GPI RAS. 2009.

9. VV Apollonov. High power lasers for space debris elimination, HPLS@A-2012, Istanbul, Turkey. 2012.

10. VV Apollonov, Pletnev NV. Formation of extended conducting channels in atmosphere. QE. 2012; 42:

11. VV Apollonov, Pletnev NV. Formation of extended Directional Breakdown Channels Produced by a Copper Wire Exploding in the atmosphere. Technical Physics. 2013; 58.

12. Su HT, Hsu RR, Chen AB, Wang YC, Hsiao WC, et al. Gigantic jets between a thundercloud and the ionosphere. Nature. 2003; 423: 974-976. PubMed: https://pubmed.ncbi.nlm.nih.gov/12827198/ 